Militßry Technical College

Kobry Elkobbah, Cairo, Egypt

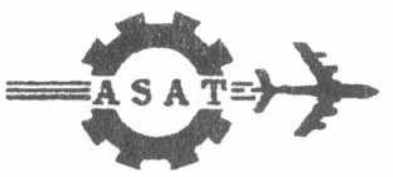

$8^{\text {th }}$ International Conference on Aerospace Sciences \&

Aviation Technology

\title{
ADAPTIVE TRACKING OF THE AIRCRAFT TRAJECTORY USING THE GCA SYSTEM
}

\author{
E. A. SOLEIT", M. E. GADALLAH"*, M. D. KHEDER
}

\section{ABSTRACT}

\begin{abstract}
A proposal of an adaptive tracking of the aircraft trajectory during the approach and landing maneuver based on the ground controlled approach (GCA) system (SR and PAR radars) is introduced. The aircraft location in polar coordinates is measured using the GCA system. However, the measured data is corrupted with noise or interference signals. Hence, the measured data is optimally estimated using a Kalman estimator. The estimated position of the aircraft is subtracted from an assigned reference path that is previously stored in the proposed GCA computer or processor. The resulting deviation error in both the elevation and the azimuth planes are encoded and transmitted to the aircraft using an up data link. The received deviation error signals are decoded and are applied to the aircraft controller to modify and adjust the aircraft control surfaces (rudder and elevator) to follow the assigned reference path. The performance of the proposed tracking system is evaluated and measured through the computer simulation.
\end{abstract}

\section{1- INTRODUCTION}

The automatic tracking of the aircraft path during the approach and landing maneuver is of great interest during the third category where the weather conditions are very bad and the runway visibility may be zero. Hence an automatic tracking of the aircraft is necessary for night flight or in the case of very poor weather conditions $[1,2]$.

* Ass. Prof., Military Technical College

** Ass. Prof., Military Technical College

*** Ph. D. Student, Military Technical College 
In this paper, a proposal for an adaptive tracking system of the aircraft during the approach and landing maneuver for both the longitudinal and the lateral motions is presented. This system includes GCA models (GCA elevation and GCA azimuth models ), Kalman estimator, the aircraft control servo and the aircraft dynamic surfaces as depicted in Figure 1.

The GCA system is responsible to measure the aircraft path during the approach and landing maneuver. A state space model of the GCA systern is derived as function of the aircraft and the controller parameters. The GCA model is an essential module to execute the optimal Kalman estimator. The performance of the aircraft path tracking model in the lateral and longitudinal motions are evaluated and tested at different signal to noise ratios through the computer simulation.

The proposed adaptive tracking of the aircraft presents smaller adaptation noise and it also converges faster to the desired path. Consequently, the proposed system can provide a high tracking speed to follow an assigned trajectory during the approach and landing maneuver.

\section{2-AIRCRAFT PATH TRACKING}

The basic problem in an aircraft motion analysis (AMA) is to estimate the trajectory of an aircraft from a noise corrupted sensor data [3]. The performance of the AMA algorithm is simplified by the motion analysis in two planes; $x-z$ and $x-y$ planes.

The longitudinal motion of the aircraft is described by two main parameters; the pitch angle $(q)$ and longitudinal velocity $\left(v^{q}\right)$. The GCA radar measures the aircraft position coordinates in the space as the elevation angle $(\mathrm{g})$ and range $(r)$ with respect to the touch down point during the landing maneuver. The lateral motion of aircraft is described by two parameters the yaw angle (f) and lateral velocity $\left(v^{f}\right)$ during approach maneuver. The aircraft position (azimuth angle $y$ and range $r$ ) is measured by the GCA radar. An adaptive closed loop system is proposed to control the aircraft longitudinal motion in the vertical plane without any action from the pilot $[4,5]$. A proposal of a tracking loop is depicted in Figure 1. It is apparent that the proposed model consists of four principal modules; the controller, the aircraft dynamics, GCA system and the Kalman estimator. The path, $p_{r}$ represents the reference landing path. For the automatic landing operation, the aircraft has to follow a reference landing which is determined previously by both the reference elevation angle $g_{\text {ref }}$ and the reference azimuth angles $y_{\text {ref. }}$ The path, $p^{\wedge}$ represents the estimated path. The error signal, $e$ is the difference between $p_{r}$ and $p^{\wedge}$ which can be expressed as :

$$
e=p_{r}-p^{\wedge}
$$

The elevation and azimuth error signals are used as input signals to the elevator and rudder controllers whose servo transfer functions can be represerited by a first order system as $[4,11,12]$ : 


$$
\begin{aligned}
& \frac{\delta_{e}(Z)}{\varepsilon_{\gamma}(Z)}=\frac{10\left(1-z^{-1}\right)}{1+0.6 Z^{-1}} \\
& \frac{\delta_{r}(Z)}{\varepsilon_{\psi}(Z)}=\frac{10\left(1-z^{-1}\right)}{1+0.7 Z^{-1}}
\end{aligned}
$$

where $\delta_{e}, \delta_{r}$ represent the output signals of the elevator and rudder controllers respectively. Furthermore, the aircraft longitudinal and lateral dynamics can be represented using a short period approximation as $[4,11,12]$ :

$$
\begin{aligned}
& \frac{\theta(Z)}{\delta_{e}(Z)}=\frac{1}{1-0.96 Z^{-1}} \cdot \frac{-0.08644-0.025 Z^{-1}+0.06389 Z^{-2}}{1-0.4966 Z^{-1}+0.651 Z^{-2}} \\
& \frac{\phi(Z)}{\delta_{r}(Z)}=\frac{1}{1-0.97 Z^{-1}} \cdot \frac{-0.09-0.036 Z^{-1}+0.0545 Z^{-2}}{1-0.389 Z^{-1}+0.6883 Z^{-2}}
\end{aligned}
$$

where $f$ and $\theta$ represent the aircraft yaw and pitch angles respectively.



Fig. 1 A model of the aircraft tracking loop

The aircraft elevation and azimuth angles are measured by the GCA system. However, the measured angles are computed with additional noise. Hence, Kalman estimator is introduced to optimally estimate the measured elevation and azimuth angles $[6,7]$. 


\section{3-THE GCA STATE SPACE MODEL}

A state space model of the GCA system can be described as function of aircraft and its controller parameters [8]:

$$
h_{k+1}=m_{k} h_{k}+b_{k} r_{k+1}
$$

where $\mathbf{h}_{\mathbf{k}}$ is defined as the state vector that describes the variation of the aircraft elevation and azimuth angles around the reference landing path. $m_{k}$, $b_{k}$ are system parameters that are chosen arbitrarily such that the mean square error criterion is minimized. $\mathbf{r}_{\mathbf{k}+1}$ represents the aircraft dynamics angles. They are expressed as:

$$
\begin{aligned}
& h_{k}=\left[g_{k} y_{k}\right] \\
& r_{k+1}=\left[q_{k+1} f_{k+1}\right]
\end{aligned}
$$

It is apparent that eq. (6) expresses the dynamic variation of the elevation and azimuth angles in accordance with the variation in the pitch and yaw angles. Hence, the measured path of the aircraft using the GCA system can be written as:

$$
p_{m}(k)=p_{r e f}+h_{k}
$$

where $p_{\text {ref }}$ represents the assigned path of the landing maneuver [2].

\section{4- KALMAN ESTIMATOR MODEL}

An adaptive Kalman estimator is used in the tracking system to estimate the aircraft path from noisy observations measured by the GCA system. The estimated path $p^{\wedge}(k)$ can be expressed in terms of the Kalman gain and the measured error signal as $[9,10]$ :

$$
p^{\wedge}(k)=p^{\sim}(k)+G(k)\left[p_{m}(k)-H p^{\sim}(k)\right]
$$

where $G(k)$ is known as the Kalman gain that is defined by :

$$
G(k)=p_{e}(k) H^{\top}(k)\left[H(k) p_{e}(k) H^{\top}(k)+R(k)\right]^{-1}
$$

where $p(k)$ is known as an error covariance that is given by:

$$
P_{e}^{+}(k)=[1-G(k) H(k)] P_{r}(k)
$$

where

$$
\begin{aligned}
& \mathrm{p}^{-}=\text {a priori estimated aircraft path } \\
& \mathrm{p}^{\wedge}=\text { a posterior estimated aircraft path } \\
& \mathrm{p}_{\mathrm{m}}=\text { a measured aircraft path } \\
& \mathrm{p}_{e}=\text { a priori Kalman filter error covariance } \\
& \mathrm{p}_{\mathrm{e}}^{+}=\text {a posterior Kalman filter error covariance } \\
& \mathrm{H}=\text { measurement parameter } \\
& \mathrm{R}=\text { measurement noise covariance parameter }
\end{aligned}
$$




\section{5- SIMULATION RESULTS}

The performance of the proposed tracking schemes depicted in Figure 1 is analyzed and evaluated through the computer simulation in the lateral and longitudinal motions. The coefficients $m_{k}$ and $b_{k}$ are optimally selected such that the mean square error is minimized. Thus, we chose $m_{k}=0.75$ and $b_{k}=$ 0.1 as simulation parameters. The simulation results in the lateral and longitudinal motions are explained in the following sections.

\subsection{The longitudinal motion}

The performance of the proposed aircraft longitudinal tracking scheme depicted in Figure 1 is analyzed and evaluated through the computer simulation. The elevation angle observation is measured during the landing maneuver according to equations (6) and (9). The aircraft start descent flight with an initial glide path angle equal to 0.07 radian and landing speed is assumed as $50 \mathrm{~m} / \mathrm{sec}$. The assigned reference elevation angle with respect to the runway axis is equal to 0.05 radian. Figures 2, 4 and 6 demonstrate the elevation angle variation versus the discrete time at different signal to noise ratio (SNR) of $5 \mathrm{~dB}, 10 \mathrm{~dB}$, and $15 \mathrm{~dB}$ respectively. It is clear that the proposed system with Kalman estimator exhibits better performance than that without Kalman estimator at input SNR of 5,10, and $15 \mathrm{~dB}$. Also, the transient and the steady state response of the proposed system with Kalman estimator is demonstrated by the system learning curves depicted in Figures 3,5 , and 7. It is observed that the error squares converge to zero after 40 samples. Furthermore; the proposed system gives an improvement 11,10 , and $9 \mathrm{~dB}$ as the output SNR to input SNR ratio at input SNR of 5,10 , and $15 \mathrm{~dB}$ respectively. It is concluded that the proposed aircraft path tracking scheme during the longitudinal motion presents smaller adaptation noise and converges faster to the desired glide path angle. Consequently, the proposed system can provide a high tracking speed to follow an assigned trajectory during the approach and landing maneuver.

\subsection{The lateral motion}

The azimuth angle observation is measured during the approach and landing maneuver using the GCA system according to equations (6) and (9). The initial aircraft azimuth angle is equivalent to $y=0.04$ radian and the aircraft approach speed is assumed $200 \mathrm{~m} / \mathrm{sec}$.

Figure 8 , demonstrate the azimuth angle variation versus the discrete time at SNR of $5 \mathrm{~dB}$. It is clear that the proposed system with Kalman estimator exhibits better performance than that without Kalman estimator. Also, the transient and the steady state response of the proposed system with Kalman estimator is demonstrated by the system learning curve depicted in Figure 9. It is observed that the squared errors converge to zero after 40 samples. 


\section{6-CONCLUSION}

It is concluded that the proposed adaptive tracking systern of the aircraft during the approach and landing maneuver for longitudinal and lateral motions exhibits a high convergence rate and small fluctuations. Also, the performance of this model depends on the accuracy of the aircraft path measurement and the SNR. The use of the Kalman estimator to estimate the aircraft path position from noisy measured data, decreases the overshoot, and converges faster to desired reference value. Also, it improves the SNR and the system stability. Moreover, a new automatic approach and landing system can be presented using the GCA system, to dispense with the human factors problems. Moreover, A state space model of the GCA system can be derived as function of the target and its controller parameters.

\section{REFERENCES}

[1] Soleit, E. A. "Landing Systems " Military Technical College, Cairo, Egypt, 1994

[2] Collinson, R. P. G "Introduction To Avionics " Chapman \& Hall, USA, 1996.

[3] Le Cadre, J. P. " Target Motion Analysis With Multiple Arrays:

Performance Analysis "IEEE, Transactions on Aerospace and Electronic Systems, Vol. 32 , No. 3, July, 1996.

[4] Robert C. N. " Flight Stability and Automatic Control" McGraw-Hill Book Company, New York, 1990.

[5] Birdsall, D. L. \& Mair, W. A. " Aircraft Performance " Cambridge University Press, USA 1996.

[6] Baheti, R. S. " Efficient Approximation Of Kalman Filter For Target Tracking" IEEE, Transaction On Aerospace And Electronic Systems, Vol. AES-22, No. 1, JAN. 1986.

[7] Osman, M. S. A. " Automatic landing system integration with GPS, INS and MLS "M.Sc Degree, Military Technical College, Cairo, Egypt, 1996.

[8] Kheder M. D. "Adaptive Tracking of The Aircraft Path During The Approach And Landing Maneuver" M.Sc Degree, Military Technical College, Cairo, Egypt, 1997.

[9] Hayes, M. H. "Statistical Digital Signal Processing And Modeling" John Wiley \& Sons, New York, 1996.

[10] Green, M.., Walls, J. \& Stensby, J. "Discrete Extended Kalman

Filter For Rizdar Pointing Error Reduction "IEEE, Transactions on Aerospace and Electronic Systems, Vol. 24, No. 1 January, 1988.

[11] Richıard J. V. " Digital Control a State Space Approach" McGraw- Hill, INC, New York, 1995.

[12] 'Boussalis and Others " Experimental Study in Adaptive Tracking Control" IEEE TA On Transaction On Aerospace And Electronic Systems, Vol. 29, No. 1, Oct. 1993. 


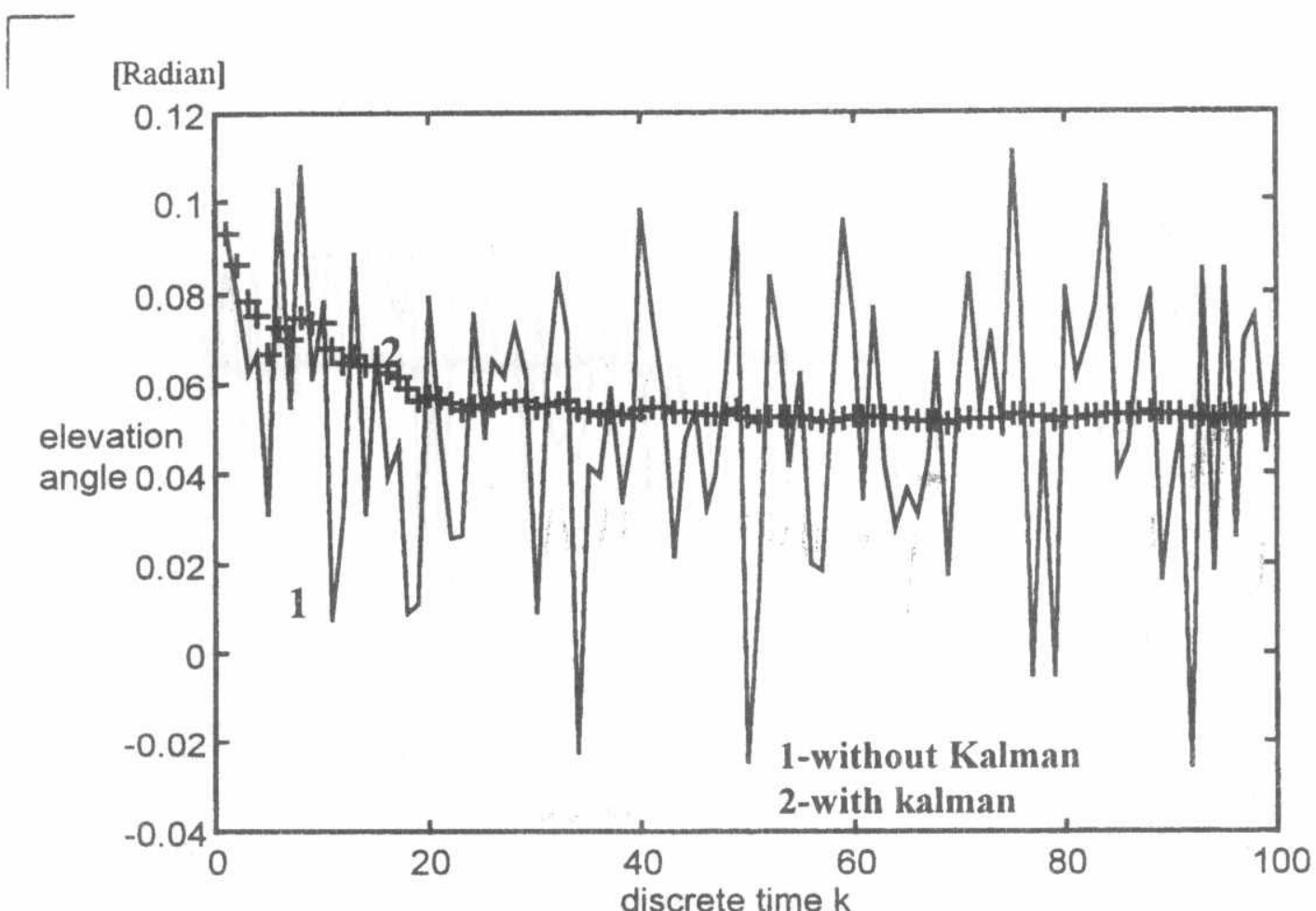

Fig. 2 The transient and steady state response of the elevation angle at SNR $5 \mathrm{~dB}$

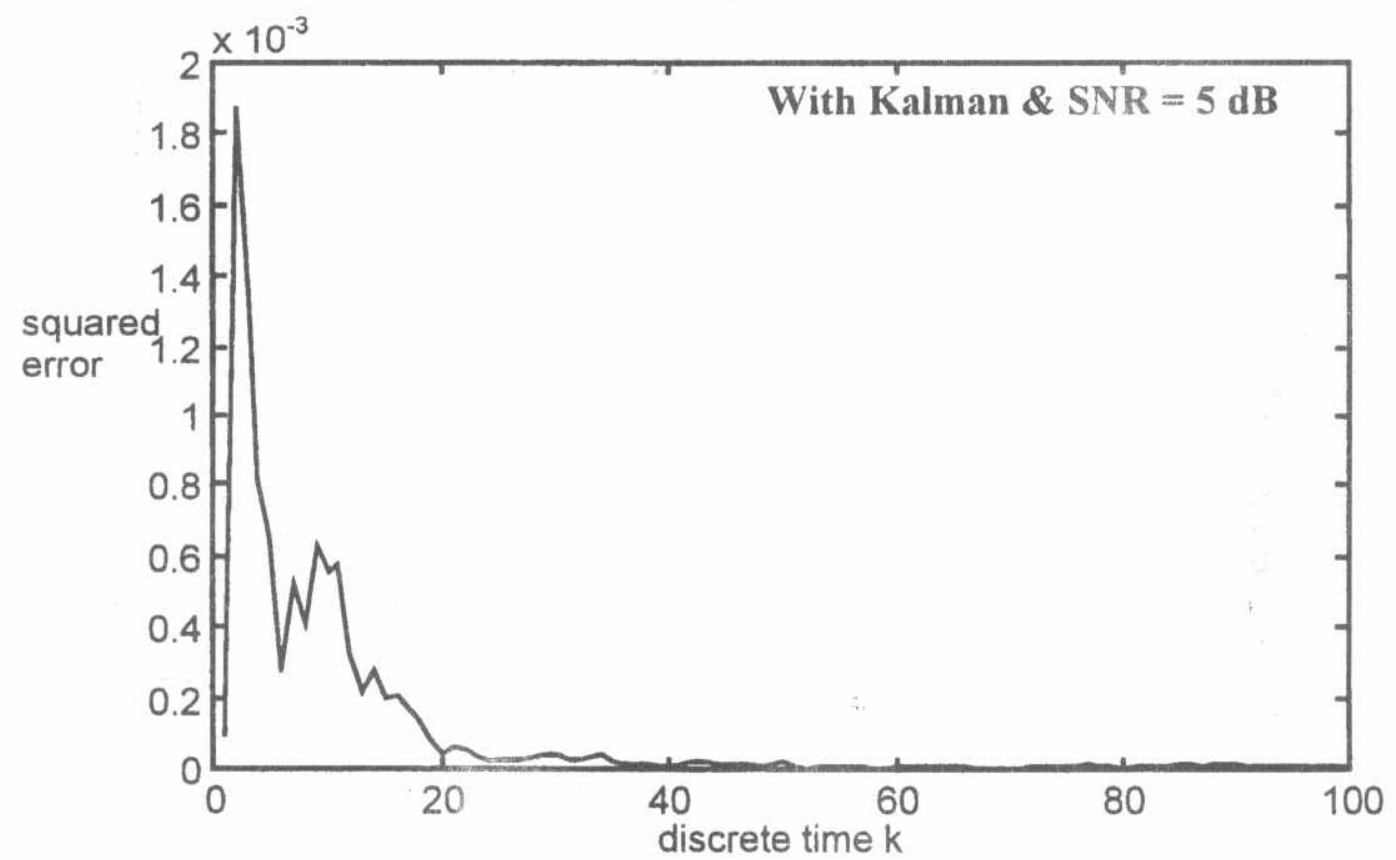

Fig. 3 The squared error of the elevation angle versus discrete time $k$ 


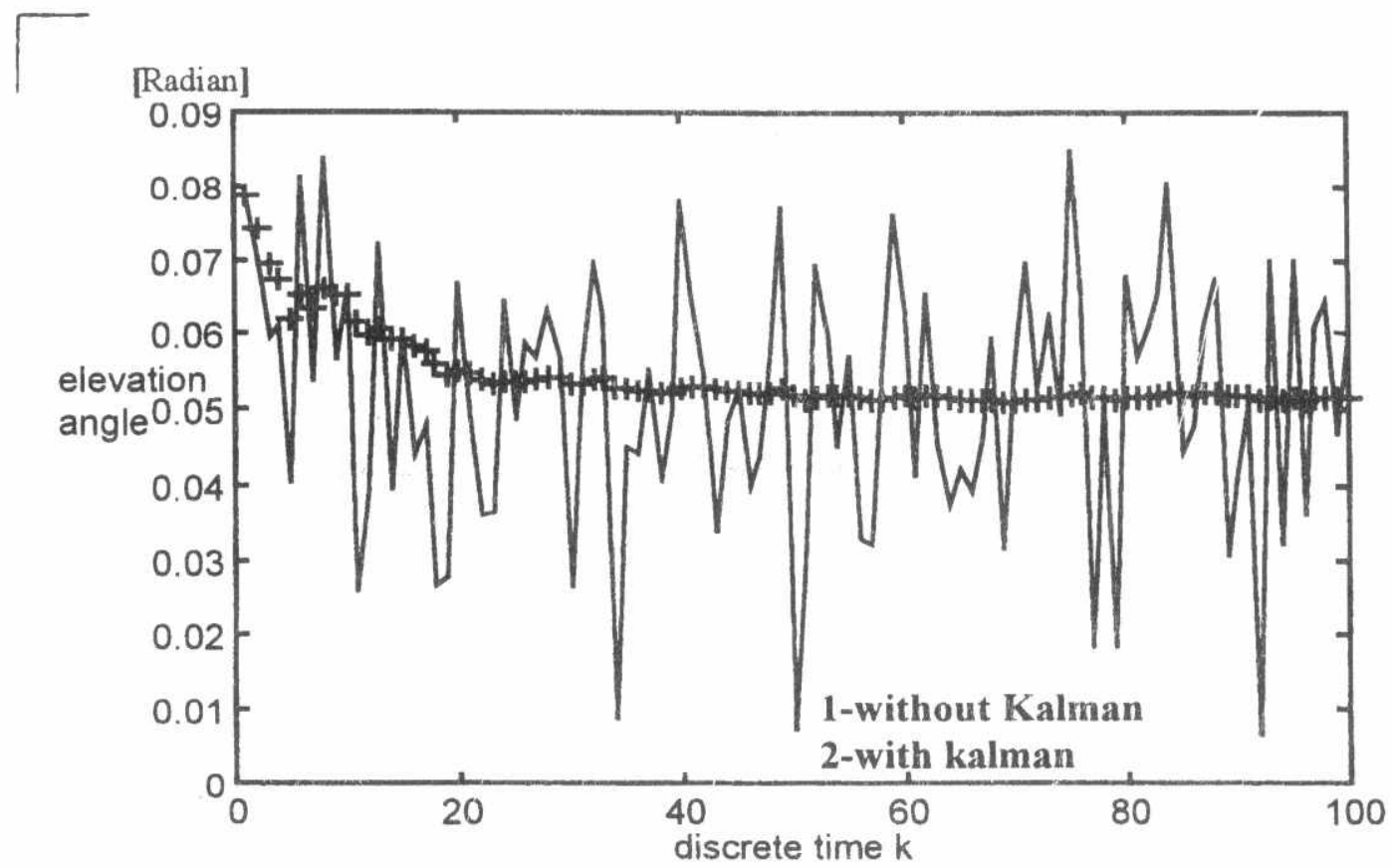

Fig. 4 The transient and steady state response of the elevation angle at SNR $10 \mathrm{~dB}$

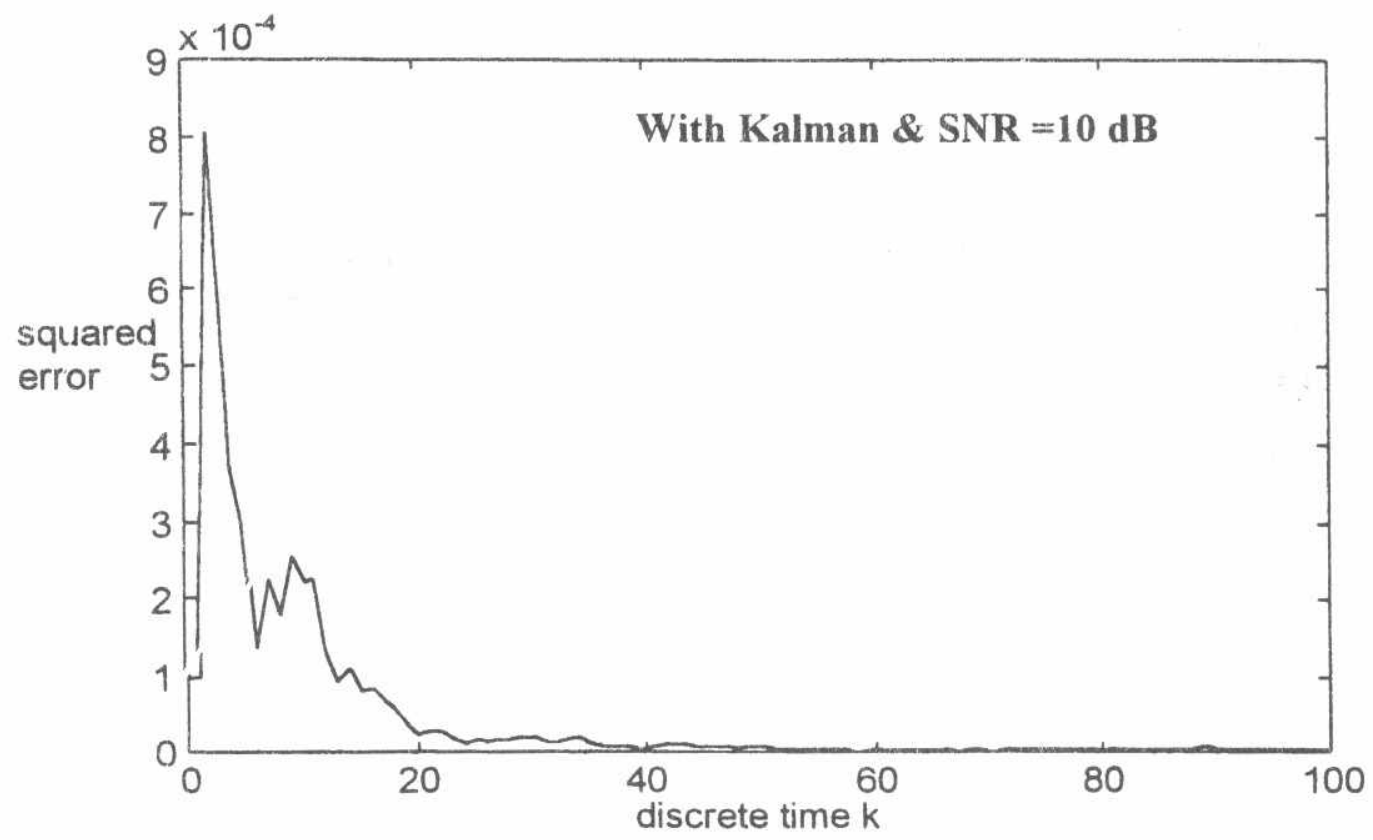

Fig. 5 The squared error of the elevation angle versus discrete time $k$ 


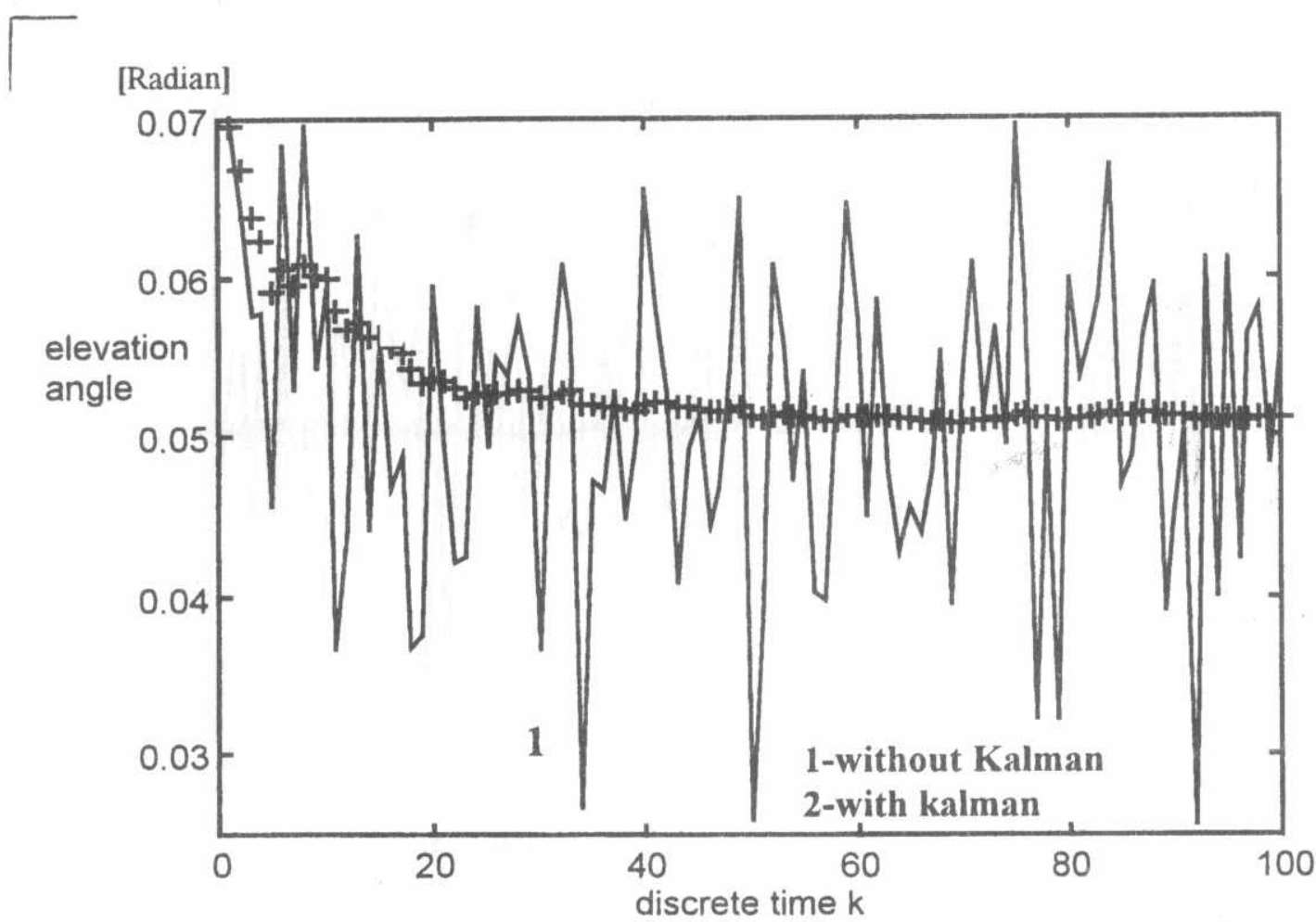

Fig. 6 The transient and steady state response of the elevation angle at SNR $15 \mathrm{~dB}$

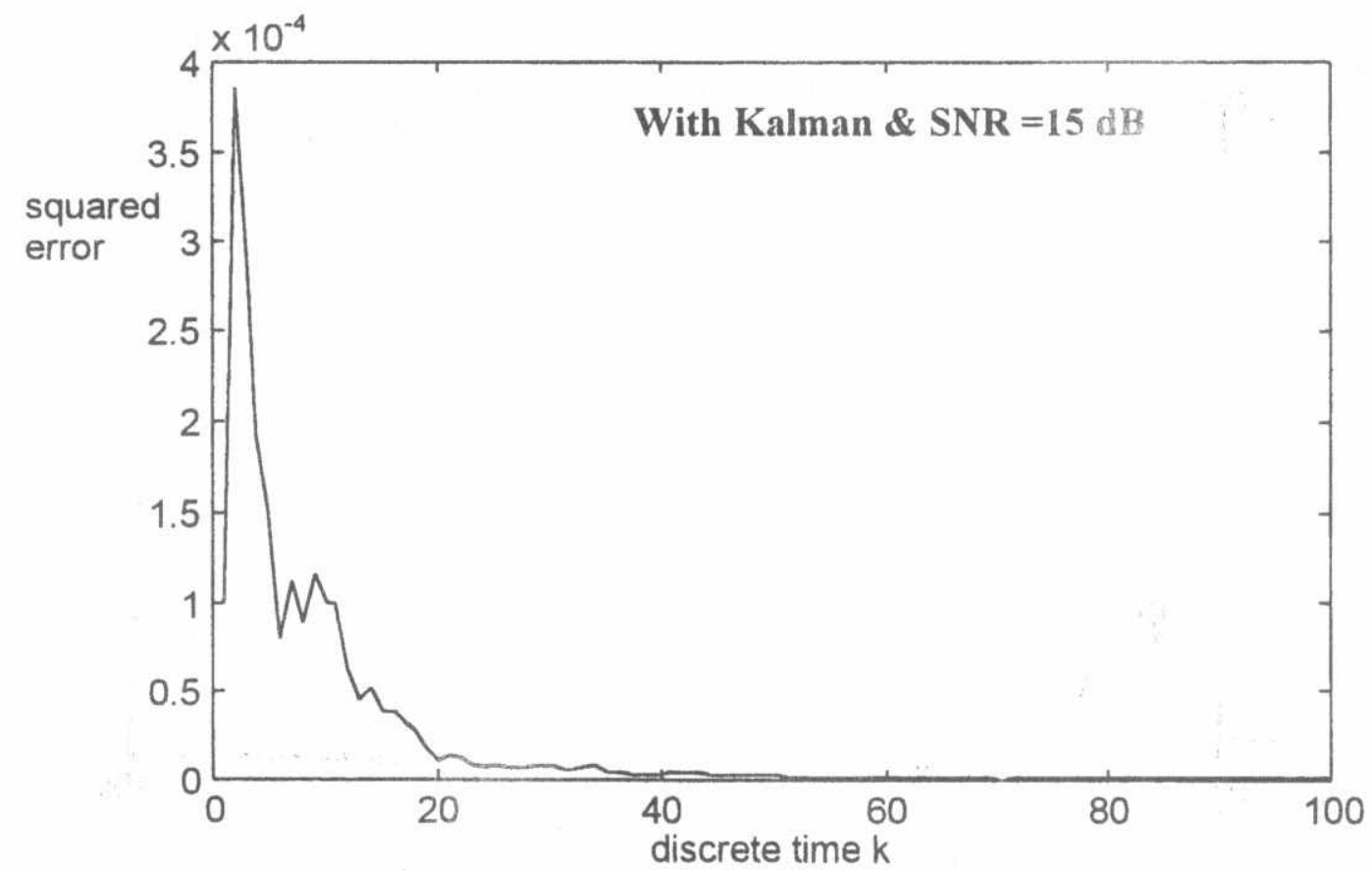

Fig. 7 The squared error of the elevation angle versus discrete time $k$ 


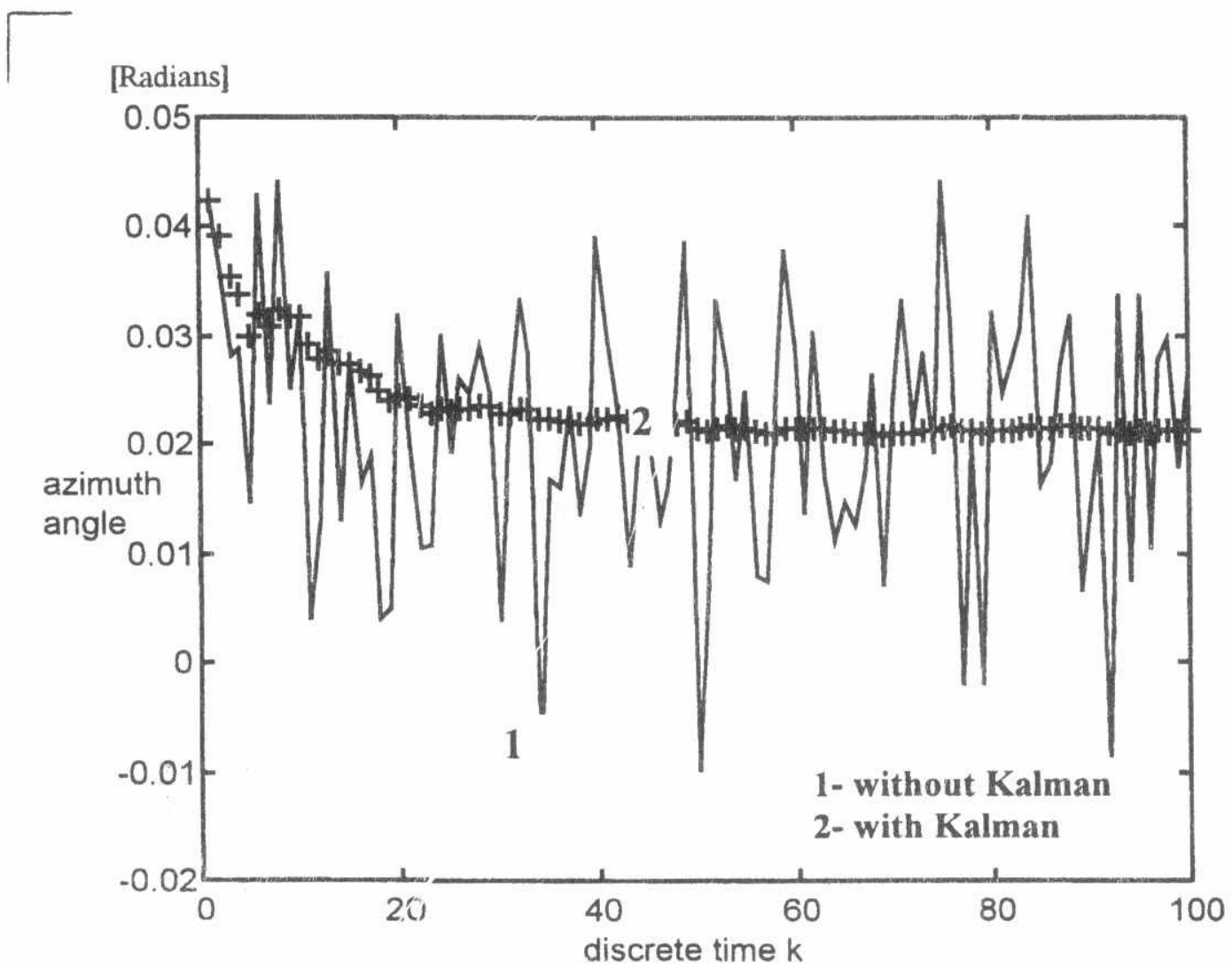

Fig. 8 The transient and steacty state response of the azimuth angle at SNR $5 \mathrm{~dB}$

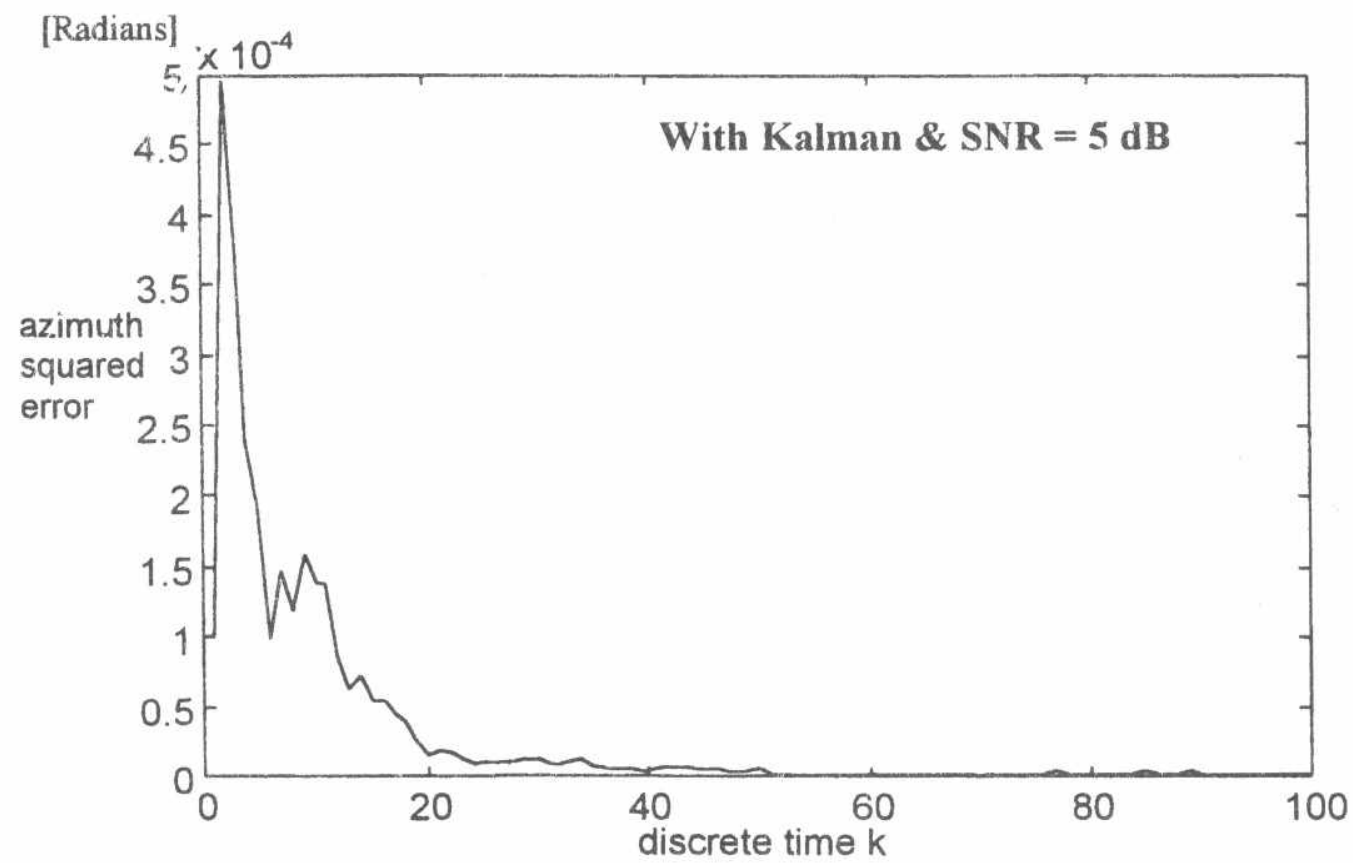

Fig. 9 The squared error of the azimuth angle versus discrete time $k$ 